\title{
Safety of benzodiazepines and opioids in interstitial lung disease: a national prospective study
}

\author{
Sabrina Bajwah ${ }^{1}$, Joanna M. Davies ${ }^{1}$, Hanan Tanash ${ }^{2}$, David C. Currow ${ }^{3}$, \\ Adejoke 0. Oluyase ${ }^{1}$ and Magnus Ekström ${ }^{2,3}$ \\ Affiliations: 'Dept of Palliative Care Policy and Rehabilitation, Cicely Saunders Institute, King's College \\ London, London, UK. ' ${ }^{2}$ ept of Respiratory Medicine and Allergology, Institution for Clinical Sciences, Lund \\ University, Lund, Sweden. ${ }^{3}$ ImPACCT, Faculty of Health, University of Technology Sydney, Sydney, Australia.
}

Correspondence: Sabrina Bajwah, Dept of Palliative Care Policy and Rehabilitation, Cicely Saunders Institute, King's College London, Bessemer Road, London, SE5 9PJ, UK. E-mail: sabrina.bajwahlakcl.ac.uk

@ERSpublications

This first ever study to examine associations between benzodiazepine and opioid use and harm in ILD supports the use of opioids and low-dose benzodiazepines in severely ill patients with respiratory compromise http://ow.ly/yBfZ30lXCKu

Cite this article as: Bajwah S, Davies JM, Tanash $\mathrm{H}$, et al. Safety of benzodiazepines and opioids in interstitial lung disease: a national prospective study. Eur Respir J 2018; 52: 1801278 [https://oi.org/ 10.1183/13993003.01278-2018].

ABSTRACT Safety concerns are a barrier to prescribing benzodiazepines (BDZs) and opioids in interstitial lung disease (ILD). We therefore examined the association of BDZs and opioids on risk of admission to hospital and death.

We conducted a population-based longitudinal cohort study of fibrotic ILD patients starting long-term oxygen therapy in Sweden between October 2005 and December 2014. Effects of BDZs and opioids on rates of admission to hospital and mortality were analysed using Fine-Gray and Cox regression while adjusting for potential confounders.

We included 1603 patients (61\% females). BDZs were used by $196(12 \%)$ patients and opioids were used by $254(15 \%)$ patients. There was no association between BDZs and increased admission. Treatment with high- versus low-dose BDZs was associated with increased mortality (subdistribution hazard ratio (SHR) $1.46,95 \%$ CI $1.08-1.98$ versus $1.13,95 \%$ CI $0.92-1.38$ ). Opioids showed no association with increased

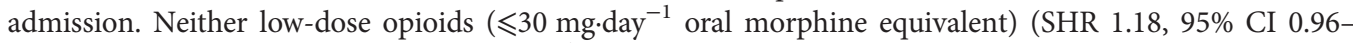
1.45) nor high-dose opioids (>30 mg.day ${ }^{-1}$ oral morphine equivalent) (SHR 1.11, 95\% CI 0.89-1.39) showed association with increased mortality.

This first ever study to examine associations between BDZ and opioid use and harm in ILD supports the use of opioids and low-dose BDZs in severely ill patients with respiratory compromise. 


\section{Introduction}

Interstitial lung disease (ILD) is the 40th most frequent cause of death globally, with a $52 \%$ increase in mortality between 2005 and 2015 [1]. Chronic breathlessness is experienced by almost all patients with advanced fibrotic ILD [2-5], and is one of the most common, burdensome and neglected symptoms affecting patients, representing a major clinical management challenge. It has a devastating impact on patients' lives, severely limiting their wellbeing and quality of life, and that of their family, friends and caregivers. It results in high health, social and informal care costs, and is one of the most frequent causes of emergency hospital admission and attendance [6]. The recent UK National Institute for Health Care and Excellence guidelines recommend the use of BDZs and/or opioids for symptomatic management of breathlessness in idiopathic pulmonary fibrosis (IPF) [7]. However, recent prospective studies showed that BDZs [8] and high-dose opioids (>30 mg.day ${ }^{-1}$ oral morphine equivalent) $[8,9]$ were associated with increased hospital admissions and deaths in patients with chronic obstructive pulmonary disease (COPD). In contrast, low-dose opioids ( $\leqslant 30 \mathrm{mg} \cdot \mathrm{day}^{-1}$ oral morphine equivalent) were not associated with hospitalisation or mortality in this group [8].

Data on the safety of BDZs and opioids in ILD are scarce, with only one relevant study where treatment outcome of 22 patients with severe interstitial pneumonia who received opioids for $24 \mathrm{~h}$ during end-of-life care was retrospectively examined [10]. 22 consecutive patients were retrospectively evaluated before and after continuous administration of opioids for $24 \mathrm{~h}$. All subjects died within 21 days; the mean survival period after opioid administration was 5.6 days. Six of the 22 patients (27\%) died within $24 \mathrm{~h}$ after opioids were initiated and hypercapnia was noted.

Ongoing concerns that these medications may cause adverse events, including respiratory depression, confusion, falls and even premature death, are cited as barriers for respiratory and palliative care health professionals. These concerns may cause reluctance to prescribe and use these medications, thus contributing to less than optimal symptomatic management, with unnecessary suffering and hospital admissions. Data on safety may help inform day-to-day management and guide clinicians on optimal management of disabling symptoms.

We therefore estimated the association of benzodiazepines (BDZs) and opioids on the risk of admission to hospital and death in patients with respiratory failure attributable to fibrotic ILD.

\section{Material and methods}

\section{Study subjects}

We included physician-diagnosed pulmonary fibrosis patients aged $\geqslant 45$ years starting long-term oxygen therapy (LTOT) between October 1, 2005 and December 31, 2014 (time period with available data on medications). Causes of pulmonary fibrosis included (but were not limited to) IPF and nonspecific interstitial pneumonia. We excluded patients who had received lung transplantation. For patients who started LTOT more than once $(n=24)$, only the most recent treatment episode was included in the analysis. Data were collected from the Swedish National Registry of Respiratory Failure (Swedevox) [11] at baseline (start of LTOT): forced expiratory volume in $1 \mathrm{~s}$ ( $\left.\mathrm{FEV}_{1}\right) \%$ pred, vital capacity (VC) \% pred, FEV1/VC, arterial oxygen tension $\left(\mathrm{PaO}_{2}\right)$ and arterial carbon dioxide tension $\left(\mathrm{PaCO}_{2}\right)$ while breathing air and oxygen, smoking status, body mass index (BMI), and World Health Organization (WHO) Performance Status.

The study was approved by the Lund University (Lund, Sweden) research ethics committee (2016/846), the Swedish National Board of Health and Welfare, and the Swedish Data Inspection Board. Individual patient consent was not required as the study used de-identified and un-re-identifiable data aggregated nationally.

\section{Study design}

This was a nationwide, prospective, population-based study of patients with physician-diagnosed oxygen-dependent fibrotic ILD in Swedevox [11]. Swedevox covers $\sim 85 \%$ of patients starting LTOT nationwide in Sweden since 1987 [11].

\section{Methods}

We obtained data on comorbidities and hospital admissions from the Swedish National Patient Register for inpatient and outpatient care for 4 years before baseline (complete data on hospitalisations since 1987 and on specialised outpatient care since 2001) [12]. Data on all dispensed prescriptions during outpatient care after July 1, 2005 were obtained from the Swedish Prescribed Drug Register. The medications of interest were categorised according to Anatomical Therapeutic Chemical codes as BDZs (N05BA), weak opioids (N02AA59 and N02AX02), strong opioids (N02A except weak), oral corticosteroids (H02A), azathioprine (L04AX01) and $N$-acetylcysteine (R05CB01). 


\section{Analysis}

Medication exposure was defined as at least one dispensed prescription during the 91 days before baseline. Exposure during follow-up was tabulated for patients classified as unexposed and exposed at baseline to evaluate whether baseline exposure was a good proxy for subsequent use during follow-up. Exposure to BDZs and opioids was coded dichotomously (treated versus nontreated), continuously as the baseline dose, and categorised into low- and high-dose treatment. The baseline dose was calculated as the mean dispensed WHO-defined daily dose (DDD) per day during the 91 days before baseline. Low-dose treatment was defined as $\leqslant 0.3 \mathrm{DDD} \cdot \mathrm{day}^{-1}$ corresponding to $\leqslant 30 \mathrm{mg} \cdot \mathrm{day}^{-1}$ oral morphine equivalent, as this is the current evidence-based dose range for opioid treatment for chronic breathlessness [13, 14]. The same cut-off was used for BDZs as it corresponded to the median dose in the exposed patients. Low-dose treatment defined as $\leqslant 0.3 \mathrm{DDD} \cdot \mathrm{day}^{-1}$ corresponded to $\leqslant 15 \mathrm{mg} \cdot$ day $^{-1}$ oral oxazepam equivalent.

BDZs (453 dispensed prescriptions) included oxazepam (87\%), diazepam (6\%) and alprazolam (5\%). Opioids included the weak opioids (281 dispensed prescriptions) tramadol (53\%) and codeine (47\%), and the strong opioids (351 dispensed prescriptions) oxycodone (49\%), morphine (25\%), fentanyl (9\%) and dextropropoxyphene (9\%).

Missing data were imputed for BMI ( $\mathrm{n}=380)$, FEV1 $(\mathrm{n}=623), \mathrm{VC}$ absolute value $(\mathrm{n}=628)$ and \% pred $(\mathrm{n}=685), \mathrm{PaO}_{2}$ on room air $(\mathrm{n}=359), \mathrm{PaCO}_{2}$ on oxygen $(\mathrm{n}=366)$, and $\mathrm{PaCO}_{2}$ on oxygen $(\mathrm{n}=249)$ using chained multiple imputation [15]. Outcome variables (days to death and days to hospitalisation) and covariates from the final models were used to estimate missing values. The number of imputations was set to 30 to reflect the amount of missing data [15] and imputed values were incorporated in the final models using Rubin's rules [15]. Covariates to be included in the final models were selected using subject matter knowledge [8]. Sensitivity analyses were conducted in people with complete data on all variables in the final models (complete case analysis) and excluding concurrent users of BDZs and opioids ( $\mathrm{n}=59(4 \%))$ (supplementary material).

Associations with the rates of hospitalisation were expressed as subdistribution hazard ratios (SHRs) estimated using Fine-Gray regression, which accounts for the competing risk of death [16]. For hospitalisations, the time under observation was from the first nonhospitalised day during LTOT until the date of first hospitalisation from all causes, with censoring at withdrawal of LTOT, death or study end (December 31, 2014). Associations with mortality were expressed as hazard ratios (HRs) and estimated using Cox regression. For mortality, the observation time was from the date of starting LTOT until the date of death from all causes, with censoring at withdrawal of LTOT or study end. The assumption of proportional hazards for the medication effects was assured using Kaplan-Meier plots and by splitting the follow-up time to examine time-specific effects. We calculated $95 \%$ confidence intervals for all estimates. Statistical analyses were performed with Stata SE version 13 (StataCorp, College Station, TX, USA). All data are reported in line with STROBE guidance [17].

\section{Results}

There was no loss to follow-up in this population-based longitudinal cohort study. We included 13 patients $(61 \%$ females) with fibrotic ILD starting LTOT. In the preceding 4 years there was a rheumatological diagnosis (any) in $7.3 \%$, pneumoconiosis in $3 \%$, sarcoidosis in $1.7 \%$ and hypersensitivity pneumonitis was present in $1.5 \%$. As shown in table 1, BDZs were used by 196 (12\%) patients and opioids by $252(16 \%)$ patients, and 59 (4\%) patients were using both BDZs and opioids. Compared with nonusers, patients taking BDZs or opioids were more likely to be female, have a lower FEV1 \% pred, worse WHO Performance Status, more hospitalisations in the 91 days prior to baseline and more comorbidities. Of note, there was no difference in their baseline lung function. Exposure to BDZs and opioids throughout follow-up was higher among patients with baseline exposure, and patients unexposed at baseline had low rates of exposed time during subsequent follow-up (table 2).

\section{Admission to hospital}

Overall, treatment with BDZs had no significant association with increased rates of hospitalisation (SHR 1.21, 95\% CI 1.00-1.46). Further analysis of low-dose BDZs ( $\leqslant 15 \mathrm{mg} \cdot \mathrm{day}^{-1}$ oral oxazepam equivalent; SHR 1.19, 95\% CI 0.95-1.49) and high-dose BDZs (>15 mg.day ${ }^{-1}$ oral oxazepam equivalent; SHR 1.27, 95\% CI 0.92-1.73) showed no association with hospitalisation. Opioids were not associated with hospitalisation (SHR 1.14, 95\% CI 0.96-1.35). Low-dose opioids ( $\leqslant 30 \mathrm{mg} \cdot \mathrm{day}^{-1}$ oral morphine equivalent; SHR 1.21, 95\% CI 0.96-1.52) and high-dose opioids (>30 mg.day ${ }^{-1}$ oral morphine equivalent; SHR 1.08 , 95\% CI 0.86-1.35) did not increase the rate of admission. There were no dose-response relations (table 3 and figure 1). 
TABLE 1 Baseline characteristics according to treatment status of 1603 patients with oxygen-dependent fibrotic interstitial lung disease

\begin{tabular}{|c|c|c|c|c|}
\hline & BDZs & Opioids & Unexposed & p-value \\
\hline Subjects & 196 & 252 & 1214 & \\
\hline Age at starting LTOT years & $77.5 \pm 8.2$ & $76.1 \pm 8.9$ & $76.3 \pm 8.8$ & 0.156 \\
\hline Female & $89(45.4)$ & $123(48.8)$ & 415 (34.2) & $<0.001$ \\
\hline FEV $_{1} \%$ pred $^{\#}$ & $66.3 \pm 37.3$ & $67.0 \pm 39.7$ & $71.6 \pm 40.3$ & 0.106 \\
\hline VC \% pred & $54.7 \pm 34.2$ & $56.2 \pm 35.6$ & $60.4 \pm 34.7$ & 0.052 \\
\hline FEV $1 / V^{\#}$ & $0.9 \pm 0.3$ & $0.9 \pm 0.3$ & $0.8 \pm 0.4$ & 0.427 \\
\hline $\mathrm{PaO}_{2}$ breathing air ${ }^{\#} \mathrm{kPa}$ & $6.5 \pm 1.0$ & $6.6 \pm 1.0$ & $6.6 \pm 1.0$ & 0.441 \\
\hline $\mathrm{PaCO}_{2}$ breathing air ${ }^{\#} \mathrm{kPa}$ & $5.3 \pm 1.0$ & $5.3 \pm 1.0$ & $5.1 \pm 0.9$ & $<0.001$ \\
\hline $\mathrm{PaO}_{2}$ breathing oxygen ${ }^{\#} \mathrm{kPa}$ & $5.7 \pm 0.9$ & $5.7 \pm 1.0$ & $5.4 \pm 1.0$ & 0.001 \\
\hline \multicolumn{5}{|l|}{ Smoking status } \\
\hline Never-smoker & $61(31.1)$ & $66(26.2)$ & $338(27.8)$ & 0.797 \\
\hline Ex-smoker & $1(1.0)$ & $3(1.2)$ & $9(0.7)$ & \\
\hline Ex-/current smoker & $105(53.6)$ & $149(59.1)$ & 698 (57.5) & \\
\hline Missing & $30(15.31)$ & $37(14.7)$ & $178(14.7)$ & \\
\hline \multicolumn{5}{|l|}{$B M I^{\Uparrow 1} \mathrm{~kg} \cdot \mathrm{m}^{-2}$} \\
\hline$<18.5$ & $10(5.1)$ & $12(4.8)$ & $35(2.9)$ & 0.579 \\
\hline $18.5-24.9$ & $107(54.6)$ & $143(56.8)$ & 707 (58.2) & \\
\hline $25-29.9$ & $53(27.0)$ & $67(26.6)$ & $314(25.9)$ & \\
\hline$\geqslant 30$ & $26(13.3)$ & $30(11.9)$ & $158(13.0)$ & \\
\hline \multicolumn{5}{|l|}{ WHO Performance Status } \\
\hline $0-1$ & 76 (38.8) & 93 (36.9) & $566(46.6)$ & $<0.001$ \\
\hline 2 & $54(27.6)$ & $70(27.8)$ & $350(28.8)$ & \\
\hline $3-4$ & $49(25.0)$ & $60(23.8)$ & $161(13.3)$ & \\
\hline Missing & $17(8.7)$ & 29 (11.5) & $137(11.3)$ & \\
\hline Hospitalisations within 4 years before baseline & $4(2-6)$ & $4(2-6)$ & $2(1-4)$ & $<0.001$ \\
\hline Hospitalised days out of the 91 days before baseline & $14(5.5-24)$ & $15(8-27)$ & $11.5(4-21)$ & $<0.001$ \\
\hline $\begin{array}{l}\text { Follow-up time hospitalised (follow-up time/ } \\
\text { hospitalised days) } \%\end{array}$ & $7.6(2.3-20.3)$ & $8.4(2.6-19.8)$ & $6.2(2.1-16.8)$ & 0.175 \\
\hline \multicolumn{5}{|l|}{ Cardiovascular diseases $\mathbf{n}$} \\
\hline 0 & $45(23.0)$ & $59(23.4)$ & 416 (34.3) & $<0.001$ \\
\hline 1 & $3(1.5)$ & $3(1.2)$ & $58(4.8)$ & \\
\hline 2 & $65(33.2)$ & $68(27.0)$ & $300(24.7)$ & \\
\hline$\geqslant 3$ & $83(42.4)$ & $122(48.4)$ & 440 (36.2) & \\
\hline \multicolumn{5}{|l|}{ Comorbidity } \\
\hline COPD & $50(25.5)$ & $67(26.4)$ & 234 (19.3) & 0.010 \\
\hline Cancer & $81(41.3)$ & $107(42.5)$ & $417(34.4)$ & 0.016 \\
\hline Depression/anxiety & $43(21.9)$ & $33(13.1)$ & $37(3.1)$ & $<0.001$ \\
\hline Diabetes mellitus & $44(22.5)$ & $73(29.0)$ & 219 (18.0) & $<0.001$ \\
\hline Injury & 33 (16.8) & $54(21.4)$ & $154(12.7)$ & $<0.001$ \\
\hline Osteoporosis & $11(5.6)$ & 35 (13.9) & 47 (3.9) & $<0.001$ \\
\hline Renal failure & $18(9.2)$ & 25 (9.9) & $65(5.4)$ & 0.007 \\
\hline Pulmonary hypertension & $12(6.1)$ & $24(9.5)$ & $86(7.1)$ & 0.315 \\
\hline GORD & $12(6.1)$ & $18(7.1)$ & $37(3.1)$ & 0.003 \\
\hline \multicolumn{5}{|l|}{ BDZs } \\
\hline High (>15 mg $\cdot$ day $^{-1}$ oral oxazepam equivalent) & 65 (33.2) & $27(10.7)$ & & \\
\hline Low ( $\leqslant 15 \mathrm{mg} \cdot$ day $^{-1}$ oral oxazepam equivalent) & $131(66.8)$ & $32(12.7)$ & & \\
\hline \multicolumn{5}{|l|}{ Opioids } \\
\hline High (>30 mg $\cdot$ day $^{-1}$ oral morphine equivalent) & $33(16.8)$ & $122(48.4)$ & & \\
\hline Low ( $\leqslant 30 \mathrm{mg} \cdot$ day $^{-1}$ oral morphine equivalent) & $27(13.8)$ & $130(51.6)$ & & \\
\hline Oral corticosteroids & $107(54.6)$ & $135(53.6)$ & $603(49.7)$ & 0.284 \\
\hline Azathioprine & $8(4.1)$ & $13(5.2)$ & 89 (7.3) & 0.142 \\
\hline $\mathrm{N}$-acetylcysteine & $81(41.3)$ & $76(30.2)$ & $372(30.6)$ & 0.010 \\
\hline
\end{tabular}

Data presented as $\mathrm{n}$, mean $\pm \mathrm{SD}, \mathrm{n}(\%)$ or median (interquartile range), unless otherwise stated. LTOT: long-term oxygen therapy; $F E V_{1}$ : forced expiratory volume in $1 \mathrm{~s}$; VC: vital capacity; $P_{\mathrm{aO}_{2}}$ : arterial oxygen tension; $\mathrm{PaCO}_{2}$ : arterial carbon dioxide tension; $\mathrm{BMI}$ : body mass index; WHO: World Health Organization; COPD: chronic obstructive pulmonary disease; GORD: gastro-oesophageal reflux disease; BDZ: benzodiazepine. Immunosuppressive drugs (including mycophenolate) used by five patients at baseline. \#: summary statistics for imputed data applying Rubin's combination rules: FEV $1 \%$ pred $(42.4 \%$ missing) VC \% pred (42.7\% missing), $\mathrm{FEV} 1$ (38.9\% missing), VC (39.2\% missing), $\mathrm{PaO}_{2}$ breathing air $(22.4 \%$ missing), $\mathrm{PaCO}_{2}$ breathing air $\left(22.8 \%\right.$ missing) and $\mathrm{PaO}_{2}$ breathing oxygen (15.5\% missing); " : missing $\mathrm{BMI}(31.1 \%)$ imputed into category $18.5-24.9 \mathrm{~kg} \cdot \mathrm{m}^{-2}$. p-value calculated using ANOVA, Kruskal-Wallis or Chi-squared test. 


\begin{tabular}{|c|c|c|}
\hline & BDZs & Opioids \\
\hline \multicolumn{3}{|l|}{ Exposed at baseline } \\
\hline Total & 196 & 252 \\
\hline Low dose & $131(66.8)$ & $130(51.6)$ \\
\hline High dose & 65 (33.2) & $122(48.4)$ \\
\hline Dose at baseline DDD.day ${ }^{-1}$ & $0.2(0.1-0.4)$ & $0.3(0.1-0.5)$ \\
\hline Exposed at baseline but unexposed during follow-up & $51(26.0)$ & $77(30.6)$ \\
\hline Exposure during follow-up $\%$ of time & $83(0-100)$ & $63(0-100)$ \\
\hline Medication-naive (unexposed) in preceding 12 months $^{\#}$ & $55(29.3)$ & $59(25.0)$ \\
\hline \multicolumn{3}{|l|}{ Not exposed at baseline } \\
\hline Total & 1407 & 1351 \\
\hline Unexposed at baseline but exposed during follow-up & $429(30.5)$ & $401(29.7)$ \\
\hline Dose during follow-up DDD $\cdot$ day $^{-1}$ & $0.0(0.0-0.0)$ & $0.0(0.0-0.0)$ \\
\hline Exposure during follow-up $\%$ of time & $0(0-16)$ & $0(0-10)$ \\
\hline Medication-naive (unexposed) in preceding 12 months ${ }^{\#}$ & $1199(92.7)$ & $1034(83.1)$ \\
\hline
\end{tabular}

Data are presented as $\mathrm{n}, \mathrm{n}(\%)$ or median (interquartile range). DDD: defined daily dose. ${ }^{\text {: }}$ among patients with at least 12 months of medication exposure data $(n=1481)$.

\section{Mortality}

In general, opioids were associated with increased mortality (SHR 1.18, 95\% CI 1.01-1.38). However, a detailed analysis of the adjusted risks for each dose level showed that neither low-dose opioids (SHR 1.22, 95\% CI 0.99-1.50) nor high-dose opioids (SHR 1.11, 95\% CI 0.89-1.39) were associated with increased mortality. BDZ treatment was related to mortality (HR 1.21, 95\% CI 1.01-1.44) and this was dose dependent. Low-dose BDZs were not associated with mortality (SHR 1.13, 95\% CI 0.92-1.38), whereas high-dose BDZs were associated with increased mortality (SHR 1.46, 95\% CI 1.08-1.97) (figures 2 and 3). As a further predictor, a WHO Performance Status of 3-4 was associated with increased mortality (SHR $2.00,95 \%$ CI $1.67-2.38$ ).

\section{Sensitivity analyses}

A complete case analysis was carried out for comparison and is shown in the supplementary material. Observed differences between the models are minor; however, in the complete case analysis, high-dose $\mathrm{BDZs}$ are no longer a significant predictor of mortality and high-dose opioids become a significant predictor of admissions. Given the much smaller sample in the complete case analysis and the bias introduced by excluding those cases with missing data, the models presented in table 3 using imputed data are more robust. When removing concurrent users of both BDZs and opioids, findings were similar to those in the main analysis (supplementary material).

\section{Discussion}

In this nationwide, population-based study using prospectively collected, linked, routine clinical data, the main findings were that treatment with opioids was not associated with increased risk of admission to hospital or death in patients with oxygen-dependent fibrotic ILD. Treatment with BDZs was not clearly associated with the rate of hospital admission, but was associated with increased mortality at higher doses.

Internationally, no medication is licensed for chronic breathlessness, but emerging evidence in other disease groups such as COPD supports regular, low-dose morphine as safe and efficacious. To the best of our knowledge, this is the first study examining safety data for BDZs or opioids in ILD. EKSTRÖM et al. [8] studied 2249 patients with oxygen-dependent COPD in Swedevox and found that low-dose opioids were not associated with increased hospital admissions or deaths. However, higher doses of opioids and all doses of BDZs were associated with increased hospital admissions and increased mortality. This is in contrast to Vozoris et al. [9], who also conducted a registry-based study and found that people with COPD prescribed opioids who had an incident opioid prescription had a 30 -day mortality risk of $1.9 \%$ compared with $1.1 \%$ without an opioid prescription after propensity score matching (i.e. an absolute difference of $0.8 \%$ ). However, it was not clear that the observed risk difference was due to opioid medication as there were many potential confounders [18]. In contrast, our study does not show an association between opioids at either low or high dose and harm. 
TABLE 3 Benzodiazepines (BDZs) and opioids and adjusted hazard ratio of admission to hospital and mortality in 1603 patients with oxygen-dependent fibrotic interstitial lung disease

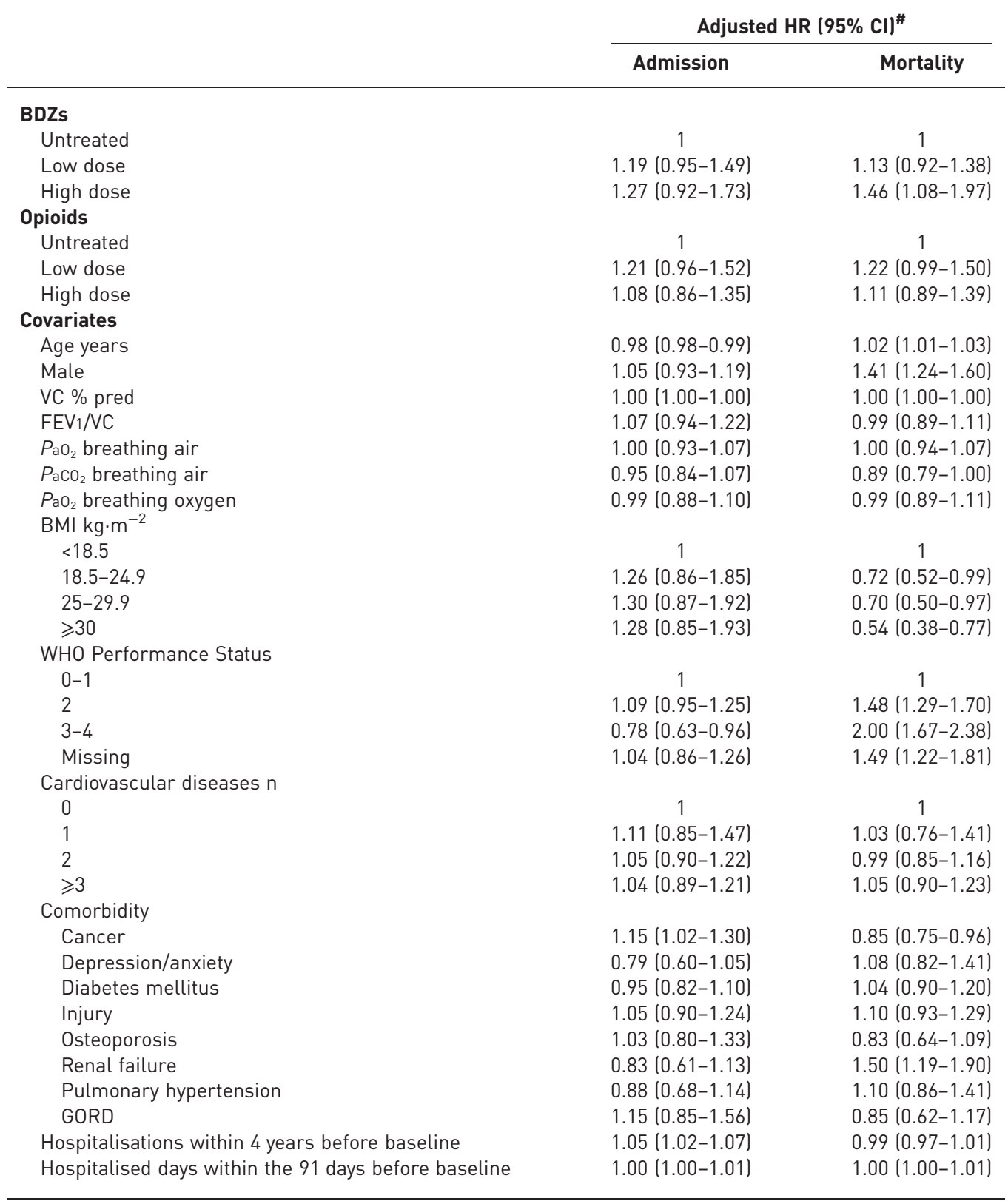

$\mathrm{VC}$ : vital capacity; $\mathrm{FEV} 1$ : forced expiratory volume in $1 \mathrm{~s} ; \mathrm{PaO}_{2}$ : arterial oxygen tension; $\mathrm{PaCO}_{2}$ : arterial carbon dioxide tension; BMI: body mass index; WHO: World Health Organization; GORD: gastro-oesophageal reflux disease. " : model adjusted for all variables in the table as well as smoking status, and use of corticosteroids, azathioprine and $\mathrm{N}$-acetylcysteine.

BDZs reduce the sensation of breathlessness through decreasing the anxiety associated with breathlessness [19]. Our data show that depressed/anxious patients were much more likely to be prescribed BDZs and opioids. Екsтвӧм et al. [8] found that all doses of BDZs were associated with increased admissions and increased mortality in their COPD patients. Our study also does not clearly show an association between BDZ use and hospital admission, but does suggest an association with mortality. As our patient group included palliative care patients, a possible explanation, at least partly, for this is that there was increased BDZ use at the end of life for management of anxiety-related breathlessness as recommended by clinical guidance [19]. Supporting this, worse WHO Performance Status was also associated with increased mortality. 
BDZs

All doses

Low dose

High dose

Opioids

All doses

Low dose

High dose

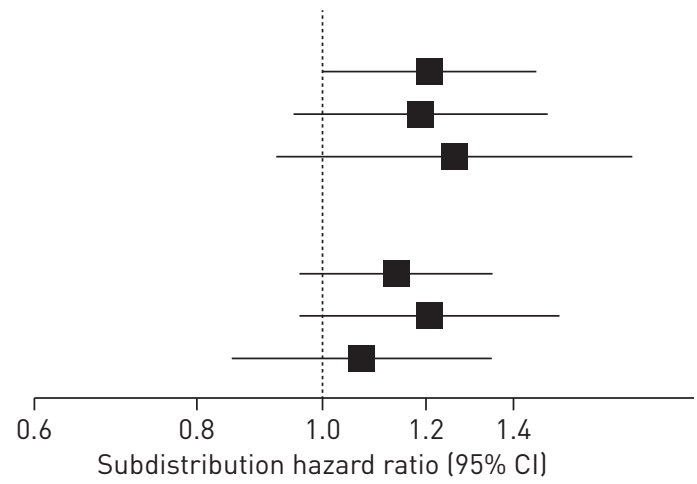

FIGURE 1 Forest plot of benzodiazepine (BDZ)/opioid treatment and hospital admissions.

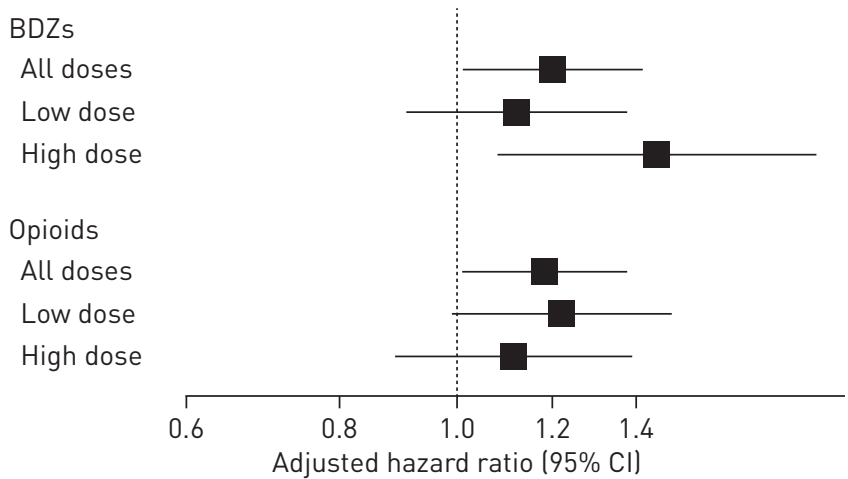

FIGURE 2 Forest plot of benzodiazepine (BDZ)/opioid treatment and mortality.

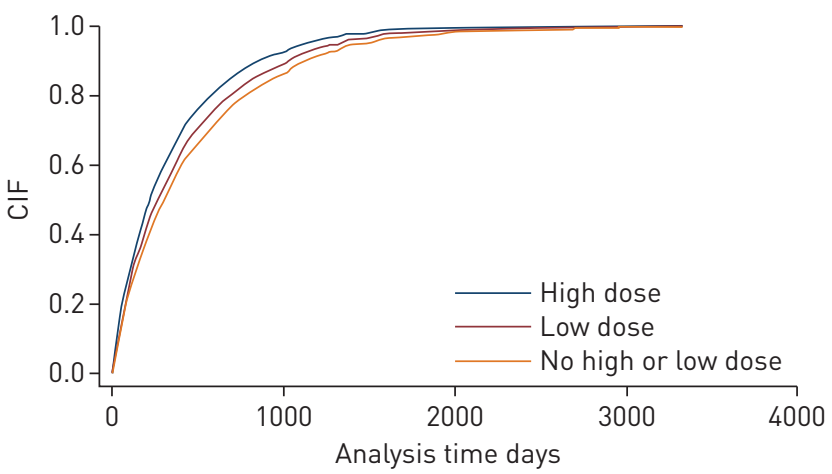

FIGURE 3 Cumulative incidence function (CIF) plot of benzodiazepine treatment on mortality.

Our data show that the use of BDZs and opioids in fibrotic ILD was low (12\% and 15\%, respectively), and that opioid prescribing was much lower than that found in a retrospective review of fibrotic ILD patients in the last year of life in the UK (18\% and 49\%, respectively) [2]. In addition, our medication-naive group (table 2) was much larger than that found by Екsтröм et al. [8]. This suggests that, when used, BDZs and opioids are not being prescribed until disease is advanced and patients are oxygen dependent. This is despite universal breathlessness at presentation $[5,20]$. This is potentially a group of patients that may benefit from holistic management of symptomatic chronic breathlessness (which is not focused on oxygen saturations), including the use of BDZs and opioids earlier in the disease process.

A recent ILD position statement stressed the importance of delivering early and effective palliative care [21]. This study provides novel data on the safety of BDZs and opioids in oxygen-dependent ILD, and may allay the fears of clinicians who have been reluctant to prescribe these medications. HigGinson et al. [22] linked 14 years of death registration data and showed that 45712 people died of ILD in England between 2001 and 2014, with increases of 9.2\% annually. Importantly, 70\% of these deaths were hospital 
deaths. Improvements in breathlessness management may decrease patient admissions and deaths, and improve the quality of life of ILD patients and carers.

Patients with ILD have a wide range of diagnoses and prognoses. Many patients can live for many years with their diagnosis and some are responsive to treatments. However, a subset of patients with progressive fibrotic ILD such as IPF has a short disease trajectory and a similar prognosis to those with lung cancer [23]. It is important to differentiate fibrotic disease in the early stages when the disease is potentially responsive to therapy [24]. However, when the disease is advanced and irreversible, this becomes less important and the focus should be on symptom control.

IPF is one of the most common ILDs. Recent European data reflects worldwide data that IPF is more common in males $(\sim 60: 40)$ and this prevalence increases with age $[25,26]$. Our data at baseline reflect a higher proportion of females $(61 \%)$. On analysis, there was a higher proportion of males in the unexposed group compared with the exposed group, indicating that females with fibrotic ILD are more likely to be prescribed BDZs and opioids. In COPD, the physiological changes affect females and males differently in terms of symptoms and quality of life. For a similar degree of physiological impairment, females experience more severe breathlessness and worse health status [27]. Females with COPD also demonstrate higher levels of anxiety and depression, and worse symptom-related quality of life, than their male counterparts [28]. Comparable data in ILD are lacking and this warrants further investigation to enable symptom management to be tailored to individual needs.

\section{Strengths and limitations of the study}

The present findings pertain to patients with severe oxygen-dependent fibrotic ILD. This is a strength of the study as this population with advanced disease and respiratory compromise may be more likely to experience adverse events. Thus, the absence of a clear association for opioid use and low-dose BDZs with hospitalisations and mortality is most informative in this population. As only 59 patients had concurrent BDZ and opioid use, we were unable to analyse the association of concurrent medications and we were also unable to examine the effect of short- versus long-acting drug forms. This was a convenience cohort, not necessarily powered to answer a question on safety, and we did not have an a priori power calculation. Therefore, we cannot exclude an effect that may be clinically significant. However, a lack of association of harm is informative. These data are comprehensive, but limited to Sweden. Data on diagnostic procedures were unavailable and therefore we are unable to present individual fibrotic ILD diagnoses. However, in a previous validation of fibrotic ILD diagnoses in the Swedevox register that included a review of medical records, $80 \%$ were classified as probable IPF [29]. Similar selection criteria were used in the present study. Recent developments of IPF registries worldwide have enabled the documentation of important parameters, including type of ILD, lung function, radiology, quality of life, ongoing treatments, follow-up and outcomes, such as death and lung transplantation [30,31]. This will make analyses of worldwide data related to ILD easier going forward. This study highlights that registry data need to be complemented with detailed pharmacovigilance randomised controlled trials quantifying adverse events and benefits to further direct clinical care.

\section{Conclusions}

To the best of our knowledge, this is the first ever study to examine associations between BDZ and opioid use and harm in ILD patients. The evidence generated indicates that opioids are not associated with harm in people with severe oxygen-dependent ILD. High-dose BDZs may be associated with increased mortality. The possibility of true causal effects, however, needs to be validated by randomised controlled trials.

By providing valuable data on the safety of BDZs and opioids, this study may allay the fears of clinicians who have been reluctant to prescribe these medications. This study supports the use of opioids and low-dose BDZs to manage breathlessness in severely ill ILD patients with respiratory compromise, i.e. patients who have significant symptom burden that impacts every part of patients' and carers' lives. Increased prescribing may lead to improved symptom control and quality of life for this patient group.

Conflict of interest: S. Bajwah has nothing to disclose. J.M. Davies has nothing to disclose. H. Tanash has nothing to disclose. D.C. Currow is an unpaid member of an advisory board for Helsinn Pharmaceuticals, is a consultant to Specialised Therapeutics and Mayne Pharma, and received intellectual property payments from Mayne Pharma. A.O. Oluyase has nothing to disclose. M. Ekström has nothing to disclose.

\section{References}

1 GBD 2015 Mortality and Causes of Death Collaborators. Global, regional, and national life expectancy, all-cause mortality, and cause-specific mortality for 249 causes of death, 1980-2015: a systematic analysis for the Global Burden of Disease Study 2015. Lancet 2016; 388: 1459-1544. 
2 Bajwah S, Higginson IJ, Ross JR, et al. Specialist palliative care is more than drugs: a retrospective study of ILD patients. Lung 2012; 190: 215-220.

3 Bajwah S, Higginson IJ, Ross JR, et al. The palliative care needs for fibrotic interstitial lung disease: a qualitative study of patients, informal caregivers and health professionals. Palliat Med 2013; 27: 869-876.

4 Johnson MJ, Yorke J, Hansen-Flaschen J, et al. Towards an expert consensus to delineate a clinical syndrome of chronic breathlessness. Eur Respir J 2017; 49: 1602277.

5 Carvajalino S, Reigada C, Johnson MJ, et al. Symptom prevalence of patients with fibrotic interstitial lung disease a systematic literature review. BMC Pulm Med 2018; 18: 78 .

6 Swigris JJ, Kuschner WG, Jacobs SS, et al. Health-related quality of life in patients with idiopathic pulmonary fibrosis: a systematic review. Thorax 2005; 60: 588-594.

$7 \quad$ National Institute for Health Care and Excellence. Diagnosis and Management of Suspected Idiopathic Pulmonary Fibrosis (CG163). 2013. www.nice.org.uk/guidance/cg163/resources/idiopathic-pulmonary-fibrosis-in-adults-diagnosisand-management-pdf-35109690087877 Date last updated: May 2017. Date last accessed: September 25, 2018.

8 Ekström MP, Bornefalk-Hermansson A, Abernethy AP, et al. Safety of benzodiazepines and opioids in very severe respiratory disease: national prospective study. BMJ 2014; 348: g445.

9 Vozoris NT, Wang X, Fischer HD, et al. Incident opioid drug use and adverse respiratory outcomes among older adults with COPD. Eur Respir J 2016; 48: 683-693.

10 Tsukuura $\mathrm{H}$, Nishimura $\mathrm{K}$, Taniguchi $\mathrm{H}$, et al. Opioid use in end-of-life care in patients with interstitial pneumonia associated with respiratory worsening. J Pain Palliat Care Pharmacother 2013; 27: 214-219.

11 Ekström M, Ahmadi Z, Larsson $\mathrm{H}$, et al. A nationwide structure for valid long-term oxygen therapy: 29-year prospective data in Sweden. Int J Chron Obstruct Pulmon Dis 2017; 12: 3159-3169.

12 Dobbins TA, Badgery-Parker T, Currow DC, et al. Assessing measures of comorbidity and functional status for risk adjustment to compare hospital performance for colorectal cancer surgery: a retrospective data-linkage study. BMC Med Inform Decis Mak 2015; 15: 55.

13 Ekström M, Nilsson F, Abernethy AA, et al. Effects of opioids on breathlessness and exercise capacity in chronic obstructive pulmonary disease. A systematic review. Ann Am Thorac Soc 2015; 12: 1079-1092.

14 Ekström M, Bajwah S, Bland JM, et al. One evidence base; three stories: do opioids relieve chronic breathlessness? Thorax 2018; 73: 88-90.

15 White IR, Royston P, Wood AM. Multiple imputation using chained equations: issues and guidance for practice. Stat Med 2011; 30: 377-399.

16 Fine JP, Gray RJ. A proportional hazards model for the subdistribution of a competing risk. J Am Stat Assoc 1999; 94: 496-509.

17 Von Elm E, Altman DG, Egger M, et al. The Strengthening the Reporting of Observational Studies in Epidemiology (STROBE) statement: guidelines for reporting observational studies. PLoS Med 2007; 4: e296.

18 Ekström M, Bajwah S, Johnson MJ. Incident opioid drug use and adverse respiratory outcomes among older adults with COPD. Eur Respir J 2017; 49: 1602311.

19 Simon ST, Higginson IJ, Booth S, et al. Benzodiazepines for the relief of breathlessness in advanced malignant and non-malignant diseases in adults. Cochrane Database Syst Rev 2016; 10: CD007354.

20 Collard HR, Pantilat SZ. Dyspnea in interstitial lung disease. Curr Opin Support Palliat Care 2008; 2: 100-104.

21 Kreuter M, Bendstrup E, Russell AM, et al. Palliative care in interstitial lung disease: living well. Lancet Respir Med 2017; 5: 968-980.

22 Higginson IJ, Reilly CC, Bajwah S, et al. Which patients with advanced respiratory disease die in hospital? A 14-year population-based study of trends and associated factors. BMC Med 2017; 15: 19 .

23 Vancheri C. Idiopathic pulmonary fibrosis and cancer: do they really look similar? BMC Med 2015; 13: 220.

24 du Bois R, King TE Jr. Challenges in pulmonary fibrosis 5: the NSIP/UIP debate. Thorax 2007; 62: 1008-1012.

25 Nalysnyk L, Cid-Ruzafa J, Rotella P, et al. Incidence and prevalence of idiopathic pulmonary fibrosis: review of the literature. Eur Respir Rev 2012; 21: 355-361.

26 Harari S, Madotto F, Caminati A, et al. Epidemiology of idiopathic pulmonary fibrosis in Northern Italy. PLoS One 2016; 11: e0147072.

27 de Torres JP, Casanova C, Hernandez C, et al. Gender associated differences in determinants of quality of life in patients with COPD: a case series study. Health Qual Life Outcomes 2006; 4: 72.

28 Di Marco F, Verga M, Reggente M, et al. Anxiety and depression in COPD patients: the roles of gender and disease severity. Respir Med 2006; 100: 1767-1774.

29 Ekström M, Gustafson T, Boman K, et al. Effects of smoking, gender and occupational exposure on the risk of severe pulmonary fibrosis: a population-based case-control study. BMJ Open 2014; 4: e004018.

30 Ferrara G, Carlson L, Palm A, et al. Idiopathic pulmonary fibrosis in Sweden: report from the first year of activity of the Swedish IPF-Registry. Eur Clin Respir J 2016; 3: 31090.

31 Behr J, Kreuter M, Hoeper MM, et al. Management of patients with idiopathic pulmonary fibrosis in clinical practice: the INSIGHTS-IPF registry. Eur Respir J 2015; 46: 186-196. 